\title{
Las estrategias de entrada en grupo en niños de primero de educación primaria
}

\author{
ENRIQUE LLIN VAÑÓ \\ al297049@uji.es \\ Clara Renau Escrig \\ al099848@uji.es \\ SARA RoselLó SeMPeRE \\ al258700@uji.es \\ CARLOS JAVIER SANCHIS LAHIGUERA \\ al295909@uji.es \\ Ghislaine Marande Perrin \\ marande@uji.es
}

\section{Resumen}

Introducción: En el presente estudio se han analizado las estrategias utilizadas por niños y niñas de primero de primaria para integrarse en un grupo de juego. Método: La muestra está formada por 115 sujetos de los que el $60,86 \%$ eran niños y $39,14 \%$ niñas. El tipo sociométrico de la muestra fue de $51,3 \%$ rechazados y $48,7 \%$ medios. La situación hipotética, guía principal del instrumento de evaluación describe a un niño o niña que entra en una clase donde están jugando dos compañeros/as suyos y él/ ella quiere jugar con ellos. Las diversas respuestas obtenidas a la pregunta «¿Qué puedes hacer para que te dejen jugar?» se organizaron en una clasificación específica de 6 tipos: agresivas, autorreferentes, recurrir a una figura de autoridad, competentes, pasivas y sin sentido. El principal objetivo del trabajo radicaba en estudiar la primera estrategia emitida por los alumnos y contrastar las respuestas en función del género y del tipo sociométrico. Se realizaron análisis descriptivos para las diversas variables; tipo de estrategias en función del género y del tipo sociométrico. Resultados: De las estrategias emitidas, la repetida con más frecuencia fue la competente (87\%). Las niñas $(95,6 \%)$ y promedios $(91,5 \%)$ utilizaron más estrategias competentes que los niños $(81,4 \%)$ y rechazados $(82,1 \%)$. Sin embargo, las diferencias en función del género y del tipo sociométrico no fueron significativas. Las escasas estrategias agresivas fueron utilizadas por los rechazados.

Palabras clave: entrada en grupo, estrategias sociales, tipo sociométrico, género, primer curso de educación primaria. 


\begin{abstract}
Introduction: In the present study we have analyzed the strategies used by first graders to join a play group. Method: The sample consisted of 115 children, $60,86 \%$ boys and $39,14 \%$ girls. Regarding the sociometric type 51,3\% were rejected and $48,7 \%$ average. The hypothetical situation utilized as main guide of the evaluation tool describes a child who comes into a class where two peers are playing and s/he wants to play with them. The distinct answers obtained to the question «What can you do to make them let you play?» were organized in a specific six-type classification: aggressive, self-referred, turning to an authority figure, competent, passive and meaningless. The main objective was to study the first strategy emitted by the children and contrast the responses by gender and sociometric type. To this aim, descriptive analyzes for the different variables were performed, such as strategies by gender and sociometric type. Results: The most often repeated strategy was of the competent type (87\%). Girls $(95,6 \%)$ and average $(91,5 \%)$ used more competent strategies than boys $(81,4 \%)$ and rejected $(82,1 \%)$. However, the differences by gender and sociometric type were not significant. The few aggressive strategies were used by the rejected.
\end{abstract}

Keywords: group entry, social strategies, sociometric type, gender, first grade primary education.

\title{
Introducción
}

En la vida cuotidiana los niños afrontan dversas situaciones sociales en diversos contextos. La mayoría de estas situaciones se dan en el contexto de los iguales. Éstas comprenden situaciones muy especificas como las que mencionan Asher y McDonald (2009) y son: entrada en grupo, la provocacion ambigua, la gestión de conficltos, escuchar, y negociar reglas.

El enfoque específico de este tipo de tareas sociales surge como alternativa a la idea de que las personas son competentes o incompetentes (Dodge, McClaskey y Feldman, 1985), es decir, se postula que la competencia social puede variar dependiendo de la situación a la que se enfrenta una persona y que una persona puede ser muy competente en determinadas situaciones y en otras no. Durante la década de 1980 se realizaron múltiples observaciones en situaciones estructuradas laboratorio (Putallaz y Gottman, 1981) o mediante situaciones hipotéticas a partir de las cuales se les pide contestar a determinadas preguntas (e.g., Dodge, 1980; Renshaw y Asher, 1983).

Una de las tareas sociales más importantes es el estudio de cómo los niños se integran en las actividades de un grupo de compañeros. Y un componente que ha captado el interés de los investigadores es conocer qué hacen los niños para intentar entrar en un grupo o las estrategias que activan para lograr este fin (Royo, 1993). Este trabajo se fundamenta a partir de la tarea social de entrada en gurpo y uno de los principales objetivos de estudio son las estrategias que que utilizan los niños en esta situación.

El principal reto que plantea esta situacion consiste en pasar de ser considerado una persona extraña a ser incluido en el gurpo. Según diversos estudios (Corsaro, 1981a y Forbes, Kantz, Paul y Lubin, 1982) existen indicadores de que cuando un niño trata de unirse en el juego a sus compañeros, tiene una probabilidad similar de ser aceptado que de encontrar la oposición de los miembros del grupo. Aunque el comportamiento de entrada en un grupo 
de iguales ha sido un tema de investigación durante más de 50 años (por ejemplo, Corsaro, 1981b; Mallay, 1935; McGrew, 1972; Washburn, 1932), la mayoría de las investigaciones que relacionan el tipo sociométrico y el comportamiento entrada han sido publicadas en la década de 1980.

Frankel (2004a) en su libro establece que la forma de interactuar de los niños/as no sigue los mismos patrones que la de los adultos y que las estrategias formales de presentación no entran en sus esquemas de relación. Algunos niños no tienen las habilidades necesarias para integrarse en un grupo de juego y por eso lo evitan, mientras que otros son muy hábiles (Frankel 2004b). Las diferentes estrategias utilizadas por los niños pueden determinar el éxito o fracaso en su intento de entrada.

Existen una gran variedad de estrategias que son utilizadas por los niños habitualmente y realizar una categorización de las mismas es necesario para poder realizar los analisis pertinentes. La clasificación que se utiliza en nuestro trabajo parte del monográfico Social Competence in Children (Dodge, Petit, McClaskey, Brown y Gottman, 1986) donde se establecen 6 tipos de estrategias: agresivas, autorreferentes, competentes, figura de autoridad, pasivas y sin sentido. A continuación se definen las estrategias que proponen:

- Agresivas, son amenazas, violencia física, irrumpir en el juego sin permiso, es decir, todo aquello que altere de forma brusca y violenta el transcurso normal del juego. En este estudio se consideraron respuestas agresivas, por ejemplo: «Les rompería las construcciones»; "cogería las piezas sin pedir permiso».

- Autorreferentes, son todas las declaraciones que hace un niño/a referidas a uno mismo. En este estudio se consideraron respuestas autorreferentes, por ejemplo: «Yo juego muy bien a las construcciones»; "sin mí no haréis una construcción bonita».

- Competentes, son peticiones corteses, declaraciones sobre el juego y comentarios amistosos. En este estudio consideramos respuestas competentes por ejemplo: «¿Puedo jugar con vosotros, por favor?»; «¡Qué construcciones más bonitas!»

- Figura de autoridad, son afirmaciones como «El maestro me ha dicho que puedo jugar», o aquellas declaraciones referentes a una persona de referencia o adulta. En este estudio se consideraron respuestas de figura de autoridad, por ejemplo: «Me "chivo" al profe»; «me iría a buscar al profesor».

- Pasivas, son comportamientos tímidos como revolotear alrededor de la zona de juego o esperar. En este estudio se consideraron respuestas pasivas, por ejemplo: «No haría nada; me esperaría».

- Sin sentido, no son coherentes o carecen de significado.

Los objetivos que se planten en esta investigación son: estudiar y clasificar la primera estrategia de entrada en grupo emitida por los niños de primero de primaria, comparar las estrategias utilizadas en función del género y comparar las estrategias utilizadas en función del tipo sociométrico.

\section{Método}

\section{Participantes}

La muestra está compuesta por 527 sujetos de primero de primaria distribuidos en 10 aulas de 4 colegios diferentes de Castellón de la Plana y Benicàssim. En cada aula se identificó la tipologia sociométrica de los alumnos, resultando: $70,7 \%$ promedios, $12,9 \%$ rechazados, $11,5 \%$ 
preferidos, 2,9 \% ignorados y $2 \%$ controvertidos. En estudio se trabajó solo con el alumnado rechazados y promedio, en total 115 sujetos ( $49,7 \%$ rechazados y $51,3 \%$ medios). Un $60,86 \%$ eran niños y un $39,14 \%$ niñas.

\section{Instrumentos}

Se utilizaron dos cuestionarios; el cuestionario sociométrico y el cuestionario de entrada en grupo. Los dos cuestionarios se pasaron individualmente y se realizaron en aulas preparadas para tal función dentro del colegio.

Cuestionario sociométrico de nominaciones entre iguales (García Bacete y González, 2010). Se trata de un sistema de nominaciones entre iguales que pone al niño en la situación de elegir a compañeros de clase en función de un criterio positivo o negativo previamente establecido. Al alumnado se le preguntó: «¿De todos los niños y niñas de tu clase con quién te gusta estar más?» y "¿De todos los niños y niñas de tu clase con quién te gusta estar menos?», para establecer las nominaciones positivas y negativas. Para identificar los tipos sociométricos se utilizó el software Sociomet (Gónzalez y García Bacete, 2010).

Tarea social de entrada en grupo. El grupo de investigación GREI (Grupo Interuniversitario de Investigación del Rechazo entre Iguales) ha elaborado un cuestionario de entrada en grupo, que consta una decripción escrita de la situacion, dos láminas de apoyo a la descripción, y 18 preguntas para analizar diversos aspectos de la competencia social (atribuciones, codificación de las señales, evaluación de las actuaciones, evaluación de las respuestas...). La historia es: «Una niño de tu edad que se llama Juan está haciendo construcciones con una amigo y se lo están pasando muy bien. Otra niño, Toni, entra en clase y los ve. Supongamos que Toni tiene muchísimas ganas de jugar a las construcciones». Existen dos versiones, una para los niños en donde los protagonistas eran chicos y otra para las niñas donde las protagonistas eran chicas.

En este estudio se analizan las respuestas que los niños proporcionan ante la situación: «Imaginate que eres Toni. "Piensa en todas las cosas que tú puedes hacer o decir para que Juan y su amigo te dejen jugar». El niño tenía la posibilidad de emitir una cantidad ilimitada de respuestas; en el caso que emitiera 2 o menos respuestas espontáneas, se le motivaba para que proporcionara más estrategias. Las respuestas se transcribían de forma literal. Posteriormente, las respuestas se codificaron según la clasificación establecida por Dodge y cols., (1986). Aunque muchos sujetos emitieron más de una respuesta, en este artículo solo se estudia la primera estrategia que los niños/as mencionarion porque consideramos que es necesario conocer el primer impulso, lo primero que los niños piensan, lo que creen que es más adecuado para ser aceptados en un grupo de juego.

\section{Resultados}

Para lograr los objetivos de este trabajo se utilizaron las técnicas de análisis de frecuencias y tablas de contingencia proporcionados por el programa estadístico SPSS versión 19.

En la tabla 1, se muestran las frecuencias de las estrategias que utilizaban los niños de primero de primaria. 
Tabla 1

Estrategias de primera respuesta

\begin{tabular}{llcc}
\hline & Frecuencia & Porcentaje válido \\
\hline \multirow{4}{*}{ Válidos } & Agresiva & 4 & 3,5 \\
& Autorreferente & 4 & 3,5 \\
& Competente & 100 & 87,0 \\
& Figura de autoridad & 2 & 1,7 \\
& Pasiva & 3 & 2,6 \\
& Sin sentido & 2 & 1,7 \\
& Total & 115 & 100,0 \\
\hline
\end{tabular}

Las 115 estrategias estudiadas fueron: 100 competentes (87\%), 4 autorreferentes (3,5\%), 4 agresivas (3,5\%), 3 pasivas (2,6 \%), 2 de figura de autoridad $(1,7 \%)$, y 2 fueron sin sentido $(1,7 \%)$.

En la tabla 2, se muestran la frecuencia de estrategias que usa cada género. En cada celda se observa la tasa de cada tipo de estrategia, el porcentaje dentro del género y el residuo corregido.

Tabla 2

Porcentajes y residuos corregidos de las estrategias utilizadas en función del género

\begin{tabular}{|c|c|c|c|c|c|c|c|c|c|}
\hline & & \multicolumn{6}{|c|}{ Tipo de estrategias } & \multirow[b]{2}{*}{ Total } \\
\hline & & & Agresiva & Autorreferente & Competente & $\begin{array}{c}\text { Figura } \\
\text { autoridad }\end{array}$ & Pasiva & $\begin{array}{l}\text { Sin } \\
\text { sentido }\end{array}$ & \\
\hline \multirow{6}{*}{ 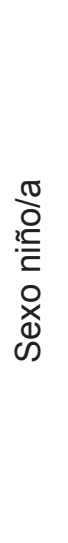 } & & $\mathrm{N}$ & 4 & 4 & 57 & 1 & 2 & 2 & 70 \\
\hline & $\stackrel{?}{\stackrel{0}{Z}}$ & $\begin{array}{l}\% \text { dentro } \\
\text { de sexo }\end{array}$ & $5,7 \%$ & $5,7 \%$ & $81,4 \%$ & $1,4 \%$ & $2,9 \%$ & $2,9 \%$ & $100 \%$ \\
\hline & & $\begin{array}{l}\text { Res. } \\
\text { corregido }\end{array}$ & 16 & 16 & -22 & $-0,3$ & 0,2 & 101 & \\
\hline & \multirow{3}{*}{$\stackrel{\mathbb{R}}{\mathrm{C}}$} & $\mathrm{N}$ & 0 & 0 & 43 & 1 & 1 & 0 & 45 \\
\hline & & $\begin{array}{l}\% \text { dentro } \\
\text { de sexo }\end{array}$ & $0 \%$ & $0 \%$ & $95,6 \%$ & $2,2 \%$ & $2,2 \%$ & $0,0 \%$ & $100 \%$ \\
\hline & & $\begin{array}{l}\text { Res. } \\
\text { corregido }\end{array}$ & $-1,6$ & $-1,6$ & 2,2 & 0,3 & $-0,2$ & $-1,1$ & \\
\hline
\end{tabular}

$\left[\mathrm{X}_{(5,115)}^{2}=7,199, p=0,206\right]$.

Los niños dijeron que utilizarían: 57 competentes (81,4 \%), 4 autorreferentes $(5,7 \%)$, 2 pasivas $(2,9 \%), 2$ sin sentido $(2,9 \%)$ y 1 figura de autoridad $(1,4 \%)$. Las niñas, dijeron que utilizarían: 43 competentes (95,6 \%), 1 pasiva (2,2 \%), 1 figura de autoridad $(2,2 \%)$, 0 sin sentido, 0 autorreferentes y 0 agresivas. 
Chicos y chicas dicen emplear las estrategias de forma diferente $\left(X^{2}{ }_{(5,115)}=7,199, p=\right.$ $0,206)$. En concreto la proporción de chicas que utilizan técnicas competentes es superior al de chicos.

En la tabla 3, se muestra la frecuencia de estrategias que se usan en función de la tipología sociométrica. En las celdas se observa la tasa de cada tipo de estrategia, el porcentaje dentro del género y el residuo corregido.

Tabla 3

Porcentajes y residuos corregidos de las estrategias utilizadas en función del tipo sociométrico

\begin{tabular}{|c|c|c|c|c|c|c|c|c|}
\hline \multicolumn{9}{|c|}{ Estrategias. Tipo de conducta de la primera respuesta } \\
\hline & & Agresiva & Autorreferente & Competente & $\begin{array}{l}\text { Figura de } \\
\text { autoridad }\end{array}$ & Pasiva & $\begin{array}{l}\text { Sin } \\
\text { sentido }\end{array}$ & Total \\
\hline \multirow{3}{*}{ 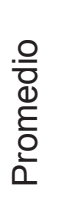 } & $\mathrm{N}$ & 0 & 3 & 54 & 1 & 1 & 0 & 59 \\
\hline & $\%$ estrategias & $0 \%$ & $5,1 \%$ & $91,5 \%$ & $1,7 \%$ & $1,7 \%$ & $0 \%$ & $100 \%$ \\
\hline & Res. corregido & $-2,1$ & 1 & 1,5 & 0 & $-0,6$ & $-1,5$ & \\
\hline \multirow{3}{*}{$\begin{array}{l}\text { 음 } \\
\mathbb{N} \\
\mathbb{N} \\
\frac{\mathbb{Z}}{0} \\
\mathbb{Q}\end{array}$} & $\mathrm{N}$ & 4 & 1 & 46 & 1 & 2 & 2 & 56 \\
\hline & $\%$ estrategias & $7,1 \%$ & $1,8 \%$ & $82,1 \%$ & $1,8 \%$ & $3,6 \%$ & $3,6 \%$ & $100 \%$ \\
\hline & Res. corregido & 2,1 & -1 & $-1,5$ & 0 & 0,6 & 1,5 & \\
\hline
\end{tabular}

$\left[\mathrm{X}_{(5,115)}^{2}=7,900, p=0,162\right]$

Los sujetos promedio utilizaron las siguientes estrategias: 54 competentes $(91,5 \%)$, 3 autorreferentes (5,1\%), 1 figura de autoridad (1,7 \%), 1 pasiva $(1,7 \%), 0$ sin sentido y 0 agresivas. Los sujetos rechazados utilizaron las siguientes estrategias: 46 competentes $(82,1 \%)$, 4 agresivas $(7,1 \%), 2$ pasivas (3,6 \%), 2 sin sentido (3,6 \%), 1 autorreferente $(1,8 \%)$ y 1 figura de autoridad $(1,8 \%)$.

Medios y rechazados dicen emplear las estrategias de forma diferente $\left[X^{2}{ }_{(5,115)}=7,900\right.$, $p=0,162]$. En concreto la proporción de medios utilizan menos técnicas agresivas que los rechazados.

\section{Discusión y conclusiones}

En los resultados se aprecia que la estrategia más utilizada en primera instancia es la competente, en un $87 \%$ de los casos. De esto se puede deducir que es la estrategia socialmente deseable, es la que todos utilizan sin pensar, es pedir amablemente que le dejen jugar. Las demás estrategias suponen una representación muy pobre con porcentajes que no superan el 3,5\%.

Es fundamental tener en cuenta las aportaciones de otros trabajos para entender mejor nuestros resultados. Los primeros trabajos que estudiaron la entrada en grupo determinaron que los niños aceptados entran partiendo del marco de referencia del grupo que está jugando y posteriormente aportan algo relevante a la actividad (Putallaz y Gottman 1981b; Putallaz, 1983). Aunque todos los niños son aceptados, rechazados e ignorados por los grupos en algún 
momento de sus intentos de entrada, los niños de tipo sociométrico bajo (rechazados, ignorados y controvertidos) tienen menos probabilidades de ser aceptados y más de ser ignorados, que los de tipologías diferentes (Putallaz y Gottman, 1981c).

Las investigaciones que correlacionan los tipos sociométricos, tradicionalmente, han sido enfocadas hacia los estilos de interacción conductual de los niños/as en los grupos de iguales (Renshaw y Asher, 1983). Una larga tradición de este tipo de investigaciones indican que los niños populares son más amistosos y constructivos en las interacciones con los iguales (e.g., Dodge, 1980; Goldman, Corsini y Urioste, 1980; Gottman, Gonso y Rasmussen, 1975; Marshall y McCandless, 1957). Los datos analizados no muestran diferencias significativas en función del tipo sociométrico, es decir, que para entrar en un grupo de iguales, los niños rechazados y promedios utilizan mayoritariamente estrategias competentes $(91,5 \%$ promedios y $82,1 \%$ rechazados). Estas diferencias no son significativas aunque los niños promedios utilizan un $9,4 \%$ más de estrategias competentes.

Los niños impopulares utilizan conductas más agresivas y disruptivas (e.g., Coie y Kupersmidt, en prensa; Dodge, in press; Hartup y cols., 1967) y comportamientos que no tienen en cuenta el marco de referencia de otros miembros del grupo. En la tabla 4 muestra que los sujetos rechazados utilizan más estrategias agresivas $(7,1 \%)$ que los medios $(0 \%)$, en la primera estrategia emitida. Los residuos corregidos indican que existen diferencias singificativas respecto las estrategias agresivas utilizadas en función del tipo sociométrico. Por tanto, podemos considerar la agresividad como una característica de las estrategias de los niños/as rechazados.

En las investigaciones de entrada en grupo se han incluido las diferencias entre sexos, y estas, no han sido significativas. Según Putallaz y Wasserman (1989), las chicas parecen más conscientes socialmente y sensibles que los chicos, por ejemplo, las niñas son más propensas a utilizar estrategias de entrada eficaces.

Nuestros resultados muestran diferencias significativas en las estrategias competentes que son, como apuntan Putallaz y Wasserman (1989b), las estrategias de entrada idóneas, las más eficaces, y las niñas $(95,6 \%)$, las utilizan más que los niños $(81,4 \%)$. Las estrategias agresivas han sido emitidas por niños, igual que las autorreferentes. Sin embargo, las diferencias de sexo en otros ámbitos (Deaux, 1985; Huston, 1983) sugieren que las niñas se socializan centradas en las demandas sociales más que los niños. En un trabajo con niños de 8 y 9 años de Brasil realizado por Souza y Rodrigues (2002), las niñas mostraron más habilidades para pedir ingresar a un juego. Los resultados de este estudio apoyan los resultados obtenidos en Brasil. En otro estudios con muestras de niños brasileros las niñas manifestaron más HHSS que ellos (Bandeira y cols., 2006; Cecconello y Koller, 2000). En definitiva, los diversos estudios apuntan que las niñas utilizan con más frecuencia estrategias competentes que los niños.

Esta investigación se fundamenta en una pequeña parte del cuestionario de entrada en grupo. Por ello, las futuras líneas de investigación son muy amplias, pero por ejemplo, Asher y cols. (1980) descubrieron que las estrategias de los niños menos populares en situaciones conflictivas eran más agresivas y que sus estrategias en situaciones de iniciación y mantenimiento tendían a ser menos sofisticadas y detalladas. Estos descubrimientos proporcionados por Asher podrían establecer una línea futura de investigación. También se podría observar si las segundas, terceras o cuartas estrategias emitidas por los niños/as mantienen la misma línea. Por otro lado, también se podría estudiar cuáles son las estrategias que utilizan los niños/as cuando se les niega la entrada en grupo.

Una de las limitaciones que de este estudio reside en la situación hipotética, es decir, que no se ha realizó una observación directa mediante una gravación. Puede que el niño no se identifique en la situación, que no le guste jugar a las construcciones y por eso no esté mo- 
tivado o familiarizado con la misma. Existen muchas variables que escapan a nuestro control y por ello, proponemos realizar la misma investigación en un contexto ecológico en el que se puedan observar las conductas directamente.

\section{Referencias bibliográficas}

Asher, S. R. y McDonald, K. L. (2009). The behavioral basis of acceptance, rejection, and perceived popularity. Handbook of Peer Interactions, 13, 232-248.

Asher, S. R., Renshaw, P. D., y Geraci, R. L. (1980). Children's friendships and social competence. International Journal of Psycholinguistics, 7, 27-39.

Bandeira, M., Rocha, S. S., Freitas, L. C., Del Prette, Z. A. P., y Del Prette, A. (2006). Habilidades sociais e variáveis sociodemográficas em estudantes do ensino fundamental. Psicologia em Estudo, 11, 541-549.

Cecconello, A. M. y Koller, S. H. (2000). Competência social e empatia: um estudo sobre resiliência com crianças em situação de pobreza. Estudos de Psicologia, 5, 71-93.

Coie, J. D., y Kupersmidt, J. B. (1983). A behavioral analysis of emerging social status in boys' groups. Child Development, 54, 1400-1416.

Corsaro, W. (1981). Friendship in the nursery school: Social organization in the peer environment. In S. Asher y J. M. Gottman (eds.). The development of children's friendships (pp. 207-241). Nueva York: Cambridge University Press.

Deaux, K. (1985). Sex and gender. Annual Review of Psychology, 36, 49-81.

Dodge, K. A. (1980). Social cognition and children's aggressive behavior. Child Development, $51,162-170$.

Dodge, K. A. (1983). Behavioral antecedents of peer social status. Child Development, 54, 1386-1389.

Dodge, K. A., McClaskey, C. L., y Feldman, E. (1985). A situational approach to the assessment of social competence in children. Journal of Consulting and Clinical Psychology, 53, 344353.

Dodge, K. A. Pettit, G. S., McClaskey, C. L., Brown, M. M. y Gottman, J. M. (1986). Social Competence in Children (1986). Monographs of the Society for Research in Child Development, 51, 1-85.

Forbes, D. L., Katz, M. M., Paul, B., y Lubin, D. (1982). Children's plans for joining play: An analysis of structure and function. En D. Forbes y M. T. Greenberg (eds.), New directions for children development: Children's planning strategies (No. 18, pp. 61-79). San Francisco: Jossey-Bass.

Frankel, F. (2010). Making Friends: Joining Others Play. En Friends forever. How parents can help their kids make and keep good friends (pp. 51-66). San Francisco: Jossey-Bass.

García-Bacete, F. J. y González, J. (2010). Evaluación de la competencia social entre iguales. Madrid: Tea Ediciones.

Goldman, J. A., Corsini, D. A., y Urioste, R. (1980). Implications of positive and negative sociometric status for assessing the social competence of young children. Journal of Applied Developmental Psychology, 1, 209-220.

Gottman, J., Gonso, J., y Rasmussen, B. (1975). Social interaction, social competence, and friendship in children. Child Development, 46, 709-718.

Hartup, W. W., Glazer, J. A., y Charlesworth, R. (1967). Peer reinforcement and sociometric status. Child Development, 38, 1017-1024. 
Huston, A. C. (1983). Sex-typing. En P. H. Mussen (Series Ed.) y E. M. Hetherington (eds.), Handbook of child psychology: Vol. 4. Socialization, personality, and social development (pp. 387-467). Nueva York: Wiley.

Mallay, H. (1935). A study of some of the techniques underlying the establishment of successful social contacts at the preschool level. Journal of Genetic Psychology, 47, 431-457.

McGrew, W. C. (1972). An ethological study of children's behavior. Nueva York: Academic Press.

Marshall, H. R., y McCandless, B. R. (1957). A study in prediction of social behavior of preschool children. Child Development, 28, 149-159.

Putallaz, M. (1983). Predicting children's sociometric status from their behavior. Child Development, 54, 1417-1426.

Putallaz, M., y Gottman, J. M. (1981). An intencional model of children's entry into peer groups. Child Development, 52, 986-994.

Putallaz, M., y Wasserman A. (1989). Children's naturalistic entry behavior and sociometric status: A developmental perspective. Developmental Psychology, 25, 297-305.

Rollo, P. (1993). El conocimiento de estrategias de interacción con los compañeros en la infancia: diferencias en función de la edad, el sexo y el grado de Aceptación Social. Tesis doctoral. Universidad Complutense de Madrid, España.

Renshaw, P. D., y Asher, S. R. (1983). Children's goals and strategies for social interaction. Merrill-Palmer Quarterly, 29, 353-374.

Souza F. y Rodrigues M. M. P. (2002). A segregação sexual na interação de crianças de 8 e 9 anos. Psicologia: reflexão e crítica, 15, 489-496.

Washburn, R. W. (1932). A scheme for grading the reactions of children in a new social situation. Journal of Genetic Psychology, 40, 84-99. 